\title{
NONEXISTENCE OF STABLE HARMONIC MAPS TO AND FROM CERTAIN HOMOGENEOUS SPACES AND SUBMANIFOLDS OF EUCLIDEAN SPACE
}

\author{
BY \\ RALPH HOWARD AND S. WALTER WEI
}

\begin{abstract}
Call a compact Riemannian manifold $M$ a strongly unstable manifold if it is not the range or domain of a nonconstant stable harmonic map and also the homotopy class of any map to or from $M$ contains elements of arbitrarily small energy. If $M$ is isometrically immersed in Euclidean space, then a condition on the second fundamental form of $M$ is given which implies $M$ is strongly unstable. As compact isotropy irreducible homogeneous spaces have "standard" immersions into Euclidean space this allows a complete list of the strongly unstable compact irreducible symmetric spaces to be made.
\end{abstract}

1. Introduction. A harmonic map is a smooth map between Riemannian manifolds which is a critical point of the energy integral. A very natural method of trying to construct harmonic maps between compact Riemannian manifolds (generalizing the classical Dirichlet principle) is to try to find a map which has least energy in its homotopy class, or more generally, is stable in the sense that it is a local minimum for the energy. For example a basic existence theorem of Eells and Sampson $[\mathbf{E S}]$ is that there is an energy minimizing (and thus harmonic) map in each homotopy class in the case the image manifold has nonpositive sectional curvatures (see [EL] for a discussion of this and other stability properties related to harmonic maps and the energy integral). Unfortunately for the existence theory in some cases there need not be any nonconstant stable harmonic maps between a given pair of compact manifolds. In particular, for the standard sphere $S^{m}$ of dimension $m \geq 3$ Xin $[\mathbf{X}]$ has shown that $S^{m}$ cannot be the domain of any nonconstant stable harmonic map and Leung $[\mathbf{L g}]$ has shown $S^{m}$ cannot be the range of any nonconstant stable harmonic map.

In this paper we will extend their methods and results to cover a larger class of manifolds. First we give a condition on the second fundamental form or Weingarten map of a compact immersed submanifold $M$ of Euclidean space which implies that $M$ is not the range or domain of any nonconstant stable harmonic map and also implies that the homotopy class of any map to or from $M$ contains elements of arbitrarily small energy. For brevity we call such a manifold strongly unstable. Then for any compact isotropy irreducible Riemannian homogeneous space $G / H$ of dimension $m$ we use the immersion into Euclidean space induced by the eigenfunctions of the first eigenvalue $\lambda_{1}$ of the Laplace-Beltrami operator on $G / H$ to show that $G / H$ is strongly unstable if and only if $m \lambda_{1}<2 \tau$, where $\tau$ is the scalar curvature of $M$. This allows us to make a list of all the compact irreducible symmetric

Received by the editors September 25, 1984 and, in revised form, March 27, 1985.

1980 Mathematics Subject Classification. Primary 58E20; Secondary 53C35.

Key words and phrases. Instability of harmonic maps, symmetric spaces. 
spaces which are strongly unstable. This last result is based on work of Smith [S] and Nagano $[\mathbf{N}]$ who made a list of the compact symmetric spaces on which the identity map is unstable. Finally we show that for any strongly unstable manifold $M$ the first two homotopy groups $\pi_{1}(M)$ and $\pi_{2}(M)$ vanish.

In what follows all maps and manifolds are assumed to be of class $C^{\infty}$ and all manifolds are assumed to be connected.

\section{Notation and statement of the main theorem.}

2.1. Let $M^{m}$ and $N^{n}$ be Riemannian manifolds and $f: M \rightarrow N$ a smooth map. Then we will denote the induced map between the tangent bundles $T M$ and $T N$ by either $d f$ or $f_{*}$. The length $\|d f\|$ of $d f$ is defined to be

$$
\left\|d f_{x}\right\|^{2}=\sum_{i=1}^{m}\left\|d f\left(e_{i}\right)\right\|^{2},
$$

where $e_{1}, \ldots, e_{m}$ is an orthogonal basis $T M_{x}$. The energy integral (or just the energy) of $f$ is then

$$
E(f)=\frac{1}{2} \int_{M}\|d f\|^{2} \Omega_{M}
$$

where $\Omega_{M}$ is the volume density on $M$. To insure the energy is finite it will be assumed $M$ is compact.

The smooth map $f: M \rightarrow N$ is harmonic iff for every smooth homotopy $f_{t}$ with $f_{0}=f$

$$
\left.\frac{d}{d t}\right|_{t=0} E\left(f_{t}\right)=0
$$

and $f$ is a stable harmonic map iff it is harmonic and for every smooth homotopy $f_{t}$ with $f_{0}=f$

$$
\left.\frac{d^{2}}{d t^{2}}\right|_{t=0} E\left(f_{t}\right) \geq 0
$$

(This is called weakly stable by some authors.)

Now assume $M^{m}$ is isometrically immersed in the Euclidean space $\mathbf{R}^{m+k}$. Let $\bar{\nabla}$ be the standard flat connection on $\mathbf{R}^{m+k}, \nabla$ the Riemannian connection on $M$ and $h$ the second fundamental form of $M$ in $\mathbf{R}^{m+k}$ (here we follow the notation of $[\mathbf{C h}])$. These are related by

$$
\bar{\nabla}_{X} Y=\nabla_{X} Y+h(X, Y),
$$

where $X, Y$ are smooth vector fields on $M, \nabla_{X} Y$ is the component of $\bar{\nabla}_{X} Y$ tangent to $M$ and $h(X, Y)$ is the component normal to $M$. If $T^{\perp} M$ is the normal bundle of $M$ in $\mathbf{R}^{m+k}, \eta$ is a smooth section of $T^{\perp} M$ and $X$ is a smooth vector field on $M$, then the Weingarten map $A_{\eta} X$ and the connection $\nabla_{\bar{X}} \frac{1}{\eta}$ in the normal bundle are defined by

$$
\bar{\nabla}_{X} \eta=-A_{\eta} X+\nabla_{X}^{\frac{1}{X}} \eta,
$$

where $-A_{\eta} X$ is the component tangent to $M$ and $\nabla_{X}^{\frac{1}{X}} \eta$ is normal to $M$. The tensors $h$ and $A$ are related by

$$
\left\langle A_{\eta} X, Y\right\rangle=\langle h(X, Y), \eta\rangle,
$$


where $X$ and $Y$ are tangent to $M$ and $\eta$ is normal to $M$. The map $h(X, Y)$ is symmetric in $X$ and $Y$ and for each $\eta$ the linear map $A_{\eta}$ is selfadjoint.

For each $x \in M$ let $e_{m+1}, \ldots, e_{m+k}$ be an orthonormal basis for the normal space $T^{\perp} M_{x}$ to $M$ at $x$. Define a selfadjoint linear map $Q_{x}^{M}: T M_{x} \rightarrow T M_{x}$ by

$$
Q_{x}^{M}=\sum_{\alpha=m+1}^{m+k}\left(2 A_{e_{\alpha}}^{2}-\operatorname{tr}\left(A_{e_{\alpha}}\right) A_{e_{\alpha}}\right) .
$$

Here $\operatorname{tr}\left(A_{e_{\alpha}}\right)$ is the trace of the linear map $A_{e_{\alpha}}$. We can now state our main result. Its proof will follow in the next two sections.

2.2 THEOREM. Let $M^{m}$ be a compact Riemannian manifold isometrically immersed in $\mathbf{R}^{m+k}$ and assume that $Q_{x}^{M}$ is negative definite at each point $x$ of $M$ (i.e. $\left\langle Q^{M} X, X\right\rangle<0$ for all $X \neq 0$ ). Then

2.2.1. (a) For every compact Riemannian manifold $N$ there are no nonconstant stable harmonic maps $f: N \rightarrow M$. (b) Moreover the homotopy class of any map from $N$ to $M$ contains elements of arbitrarily small energy.

2.2.2. (a) For every Riemannian manifold $\tilde{M}$ there are no nonconstant stable harmonic maps $f: M \rightarrow \tilde{M}$. (b) Moreover the homotopy class of any map from $M$ to $\tilde{M}$ contains elements of arbitrarily small energy.

2.3 REMARK. In the case $M$ is the standard sphere $S^{m}$, then 2.2.2(a) is due to Xin $[\mathbf{X}]$ and in the case $M$ is a sufficiently convex hypersurface in a Euclidean space, 2.2.1(a) is due to Leung $[\mathbf{L g}]$. Both the proofs in their papers and the proofs here are based on an ingenious averaging argument introduced in the paper $[\mathbf{L S}]$ of Lawson and Simons.

2.4 DEFINITION. Let $M$ be a compact Riemannian manifold with metric $g$. Then call the metric $g$ strongly unstable if $(M, g)$ satisfies the four conditions 2.2.1(a), (b) and 2.2.2(a), (b). If the metric is clear from context the manifold $M$ will be referred to as strongly unstable.

It is elementary but still rather surprising that 2.2.1(b) and 2.2.2(b) are preserved under a change of metric on $M$.

2.5 Proposition. Let $M$ be a compact Riemannian manifold with a strongly unstable metric $g_{0}$. Then for any other Riemannian metric $g$ on $M$,

(A) for any compact Riemannian manifold $(N, h)$ the homotopy class of any map from $(N, h)$ to $(M, g)$ contains elements of arbitrarily small energy,

(B) for any Riemannian manifold $(\tilde{M}, \tilde{g})$ the homotopy class of any map from $(M, g)$ to $(\tilde{M}, \tilde{g})$ contains elements of arbitrarily small energy.

ProOF. This follows at once from [EL, Lemma 5.15, p. 37].

2.6 Remark. That a standard sphere of dimension at least three has property (B) of the last proposition is due to Eells and Lemaire [EL] and when $M$ is a compact simply connected Lie group it is due to Min-Oo $[\mathbf{M}]$. If the homotopy class of the map from $N$ to $M$ contains a submersion and $M$ is a standard sphere, then part (A) is due to Eells and Lemaire, but that this is true for any into a standard sphere (of dimension at least three) seems to be new.

2.7. We now give two easy corollaries to the theorem in the case $M^{m}$ is a convex hypersurface in $\mathbf{R}^{m+1}$. Recall that a compact hypersurface $M^{m}$ in $\mathbf{R}^{m+1}$ is an 
ovaloid iff there is a choice of a unit normal $\eta$ on $M$ so that $A_{\eta}$ is always positive definite; in this case order the eigenvalues of $A_{\eta}$ (which are the principal curvatures of $M$ ) so that $0<\lambda_{1} \leq \lambda_{2} \leq \cdots \leq \lambda_{m}$. The eigenvalues of $Q^{M}$ are then easily computed to be

$$
\lambda_{i}\left(2 \lambda_{i}-\left(\lambda_{1}+\cdots+\lambda_{m}\right)\right), \quad 1 \leq i \leq m .
$$

As $Q^{M}$ is negative definite if and only if all its eigenvalues are negative we have

2.8 COROLLARY. If the principal curvatures of the ovaloid $M^{m}$ satisfy

$$
\lambda_{m}<\lambda_{1}+\cdots+\lambda_{m-1},
$$

then $M^{m}$ is strongly unstable. In particular when $m \geq 3$ the standard unit sphere in $\mathbf{R}^{m+1}$ is strongly unstable.

It is of interest to see how this relates to the intrinsic geometry of $M^{m}$.

2.9 COROLLARY. If $M^{m}$ is an ovaloid which is pointwise $\delta$ pinched (that is for each $x \in M$ there is an $r(x)>0$ such that all sectional curvatures of $M$ at $x$ are in the interval $[\delta r(x), r(x)])$ for some $\delta>1 /(m-1)$, then $M$ is strongly unstable.

PROOF. As the Weingarten map is selfadjoint there is an orthonormal basis $e_{1}, \ldots, e_{m}$ so that $A_{\eta} e_{i}=\lambda_{i} e_{i}$. Let $K_{i j}$ be the sectional curvature of the two-plane spanned by $e_{i}$ and $e_{j}$. Then, by the Gauss curvature equation, $K_{i j}=\lambda_{i} \lambda_{j}$. Thus for $i<j, \delta r(x) \leq \lambda_{i} \lambda_{j} \leq r(x)$. Therefore

$$
\begin{aligned}
\lambda_{m-1} \lambda_{m} & \leq r(x)<(m-1) \delta r(x) \\
& \leq \lambda_{m-1}\left(\lambda_{1}+\cdots+\lambda_{m-2}\right)+\lambda_{m-1} \lambda_{m-2} \\
& \leq \lambda_{m-1}\left(\lambda_{1}+\cdots+\lambda_{m-2}+\lambda_{m-1}\right) .
\end{aligned}
$$

Dividing by $\lambda_{m-1}$ shows the last corollary applies.

2.10. We now show that the map $Q^{M}$ can be rewritten in several different forms which will be useful to us later. Let $R$ be the curvature tensor of $M$ with signs chosen so that $R(X, Y)=\nabla_{X} \nabla_{Y}-\nabla_{Y} \nabla_{X}-\nabla_{[X, Y]}$ and define the Ricci tensor $\operatorname{Ric}^{M}: T M_{x} \rightarrow T M_{x}$ by

$$
\operatorname{Ric}^{M}(X)=\sum_{i=1}^{m} R\left(X, e_{i}\right) e_{i},
$$

where $e_{1}, \ldots, e_{m}$ is an orthonormal basis of $T M_{x}$. Then the Gauss curvature equation implies [Ch, p. 76]

$$
\mathrm{Ric}^{M}-\sum_{\alpha=m+1}^{m+k} \operatorname{tr}\left(A_{e_{\alpha}}\right) A_{e_{\alpha}}+\sum_{\alpha=m+1}^{m+k} A_{e_{\alpha}}^{2}=0,
$$

where $e_{m+1}, \ldots, e_{m+k}$ are as in the equation (2.7) which defines $Q^{M}$. Using this in the definition of $Q^{M}$ yields

$$
\begin{aligned}
Q^{M} & =\sum_{\alpha=m+1}^{m+k}\left(2 A_{e_{\alpha}}^{2}-\operatorname{tr}\left(A_{e_{\alpha}}\right) A_{e_{\alpha}}\right) \\
& =-2 \operatorname{Ric}^{M}+\sum_{\alpha=m+1}^{m+k} \operatorname{tr}\left(A_{e_{\alpha}}\right) A_{e_{\alpha}}=-\mathrm{Ric}^{M}+\sum_{\alpha=m+1}^{m+k} A_{e_{\alpha}}^{2} .
\end{aligned}
$$


We remark that $Q^{M}$ can also be defined in terms of the second fundamental form $h$ of $M$. Let $e_{1}, \ldots, e_{m}$ be an orthonormal basis of $T M_{x}$. Then for $X \in T M_{x}$

$$
\left\langle Q_{x}^{M} X, X\right\rangle=\sum_{i=1}^{m}\left(2\left\|h\left(X, e_{i}\right)\right\|^{2}-\left\langle h(X, X), h\left(e_{i}, e_{i}\right)\right\rangle\right) .
$$

Using this last equation it is possible to prove the following result: Let $M^{m}$ be a compact submanifold of the unit sphere $S^{m+k-1}$ in $\mathbf{R}^{m+k}$ and let $B$ be the second fundamental form of $M$ in $S^{m+k-1}$. Then

$$
\begin{array}{ll}
\|B\|^{2}<\frac{4(m-2)}{\sqrt{m}+5}, & 3 \leq m \leq 8, \\
\|B\|^{2}<\frac{2(m-2)}{\sqrt{m}+1}, & 9 \leq m,
\end{array}
$$

implies $M$ is strongly unstable.

As the proof is nothing but a tedious exercise in completing the square (and the result does not seem to lead to any interesting new examples) we omit it.

2.11 REMARK. If for $1 \leq j \leq l$ we have a compact Riemannian manifold $M_{j}^{m_{j}}$ isometrically immersed in $\mathbf{R}^{m_{j}+k_{j}}$ in such a way that each $Q^{M_{j}}$ is negative definite, then it is easily checked that for the product immersion of $M^{m}=M_{1} \times \cdots \times M_{l}$ into $\mathbf{R}^{m+k}\left(m=m_{1}+\cdots+m_{l}, k=k_{1}+\cdots+k_{l}\right)$ that $Q^{M}$ is also negative definite. For example, if each $m_{j} \geq 3$, then the product of spheres $S^{m_{1}} \times \cdots \times S^{m_{l}}$ is a strongly unstable manifold.

\section{Proof of 2.2.1.}

3.1. Let $M^{m}$ be as in the statement of Theorem 2.2. For each smooth vector field $V$ on $M$ let $\varphi_{t}^{V}$ be the flow or one-parameter group of diffeomorphisms generated by $V$. Let $f: N^{n} \rightarrow M^{m}$ be a smooth map. Then we are going to compute

$$
\left.\frac{d^{2}}{d t^{2}}\right|_{t=0} E\left(\varphi_{t}^{V} \circ f\right)=\left.\int_{N} \frac{1}{2} \sum_{i=1}^{n} \frac{d^{2}}{d t^{2}}\right|_{t=0}\left\|\varphi_{t_{*}}^{V} d f\left(e_{i}\right)\right\|^{2} \Omega_{N}
$$

(where $e_{1}, \ldots, e_{n}$ is a local orthonormal moving frame on $N$ for several choices of the vector field $V$, all constructed from the immersion of $M^{m}$ into $\mathbf{R}^{m+k}$ ) and average the result to get rid of some troublesome terms.

To start define a field $\AA^{V}$ of linear transformations of tangent spaces of $M$ by

$$
\AA^{V}(X)=\nabla_{X} V \text {. }
$$

Then it is shown in [LS, Lemma 1, p. 434] (which contains a misprint; the $2\left\|A^{V} \xi\right\|^{2}$ in the formula for $\frac{1}{2} F^{\prime \prime}(0)$ should be replaced by $\left\|A^{V} \xi\right\|^{2}$ ) that for any vector $X$ tangent to $M$

$$
\left.\frac{1}{2} \frac{d^{2}}{d t^{2}}\right|_{t=0}\left\|\varphi_{t}^{V} X\right\|^{2}=\left\langle A^{V} \AA^{V} X, X\right\rangle+\left\langle A^{V} X, A^{V} X\right\rangle+\left\langle\left(\nabla_{V} \AA^{V}\right) X, X\right\rangle .
$$

Let $v$ be a vector in $\mathbf{R}^{m+k}$. Then define a field of vectors $v^{T}$ tangent to $M$ and a field of vectors $v^{\perp}$ normal to $M$ by

$$
\begin{aligned}
& v^{T}(x)=\text { orthogonal projection of } v \text { onto } T M_{x}, \\
& v^{\perp}(x)=\text { orthogonal projection of } v \text { onto } T^{\perp} M_{x} .
\end{aligned}
$$


Then as $v$ can be identified with a parallel field on $\mathbf{R}^{m+k}$ (i.e. $\bar{\nabla} v=0$ ) so using (2.4), (2.5)

$$
\begin{gathered}
\mathcal{A}^{v^{T}}(X)=\nabla_{X} v^{T}=\left(\bar{\nabla}_{X} v-\bar{\nabla}_{X} v^{\perp}\right)^{T}=A_{v^{\perp}} X, \\
\nabla_{X}^{\perp} v^{\perp}=\left(\bar{\nabla}_{X} v-\bar{\nabla}_{X} v^{T}\right)^{\perp}=-h\left(X, v^{T}\right) .
\end{gathered}
$$

These imply

$$
\left(\nabla_{v^{T}} A^{v^{T}}\right)=\left(\bar{\nabla}_{v^{T}} A\right)_{v^{\perp}}-A_{h\left(v^{T}, V^{T}\right)},
$$

where $\left(\bar{\nabla}_{v^{T}} A\right)$ is the derivative with respect to the connection of van der WaerdenBortolotti [Ch, pp. 65-66]. Putting these into (3.3) and using that $A_{v^{\perp}}$ is selfadjoint, we obtain

$$
\left.\frac{1}{2} \frac{d^{2}}{d t^{2}}\right|_{t=0}\left\|\varphi_{t}^{v^{T}} X\right\|^{2}=2\left\langle A_{v^{\perp}}^{2} X, X\right\rangle+\left\langle\left(\bar{\nabla}_{v^{T}} A\right)_{v^{\perp}} X, X\right\rangle-\left\langle A_{h\left(v^{T}, v^{T}\right)} X, X\right\rangle .
$$

For each vector $X$ tangent to $M$ at $x$ define a quadratic form $\mathcal{Q}_{X}$ on $\mathbf{R}^{m+k}$ by

$$
\mathcal{Q}_{X}(v)=\left.\frac{1}{2} \frac{d^{2}}{d t^{2}}\right|_{t=0}\left\|\varphi_{t .}^{v^{T}} X\right\|^{2}
$$

We now compute the trace of $\mathcal{Q}_{X}$. Toward this end choose an orthonormal basis $v_{1}, \ldots, v_{m+k}$ of $\mathbf{R}^{m+k}$ such that $v_{1}, \ldots, v_{m}$ is a basis of $T M_{x}$ and $v_{m+1}, \ldots, v_{m+k}$ is a basis of $T^{\perp} M_{x}$. Then for each $j\left(\bar{\nabla}_{v_{j}^{T}} A\right)_{v_{j}^{\perp}}=0$ as $v_{j}^{T}=0$ or $v_{j}^{\perp}=0$. Therefore using (3.7) in (3.8) yields

$$
\operatorname{trace}\left(\mathcal{Q}_{X}\right)=\left\langle 2 \sum_{\alpha=m+1}^{m+k} A_{v_{\alpha}}^{2} X, X\right\rangle-\left\langle\sum_{i=1}^{m} A_{h\left(v_{i}, v_{i}\right)} X, X\right\rangle .
$$

But a calculation using 2.6 shows

$$
\sum_{j=1}^{m} A_{h\left(v_{j}, v_{j}\right)}=\sum_{\alpha=m+1}^{m+k} \operatorname{tr}\left(A_{v_{\alpha}}\right) A_{v_{\alpha}} .
$$

Using this and the definition of $Q_{x}^{M}$ in (3.9) gives

$$
\operatorname{trace}\left(\mathcal{Q}_{X}\right)=\left\langle Q_{x}^{M} X, X\right\rangle \text {. }
$$

This along with (3.1), (3.7) and (3.8) prove

3.2 Proposition. Let $M, N$ and $f$ be as above (it is not assumed $f$ is harmonic) and let $v_{1}, \ldots, v_{m+k}$ be any orthonormal basis of $\mathbf{R}^{m+k}$. Then

$$
\left.\sum_{j=1}^{m+k} \frac{d^{2}}{d t^{2}}\right|_{t=0} E\left(\varphi_{t}^{v_{j}^{T}} \circ f\right)=\int_{N} \sum_{i=1}^{n}\left\langle Q^{M} d f\left(e_{i}\right), d f\left(e_{i}\right)>\Omega_{M} .\right.
$$

This easily implies 2.2.1(a). For if $f: N \rightarrow M$ is a nonconstant harmonic map and $Q^{M}$ is negative definite, then (3.11) implies $\left.\left(d^{2} / d t^{2}\right)\right|_{t=0} E\left(\varphi_{t}^{v_{j}^{T}} \circ f\right)<0$ for some $j$. Thus $f$ is not stable. 
The proof of 2.2.1(b) is based on

3.3 LEMMA. If $Q_{x}^{M}$ is negative definite at all points $x \in M$, then there is a number $0<\rho<1$ such that for any compact manifold $N$ and any smooth $f: N \rightarrow M$ there is a map $f_{1}: N \rightarrow M$ homotopic to $f$ with $E\left(f_{1}\right) \leq \rho E(f)$.

Once this is proven then 2.2.1(b) is straightforward. Start with any $f: N \rightarrow M$ and use the lemma to find $f_{1}$ homotopic to $f$ with $E\left(f_{1}\right) \leq \rho E(f)$. Another application of the lemma gives an $f_{2}$ homotopic to $f_{1}$ (and thus to $f$ ) with $E\left(f_{2}\right) \leq$ $\rho E\left(f_{1}\right) \leq \rho^{2} E(f)$. By induction there is an $f_{l}(l=1,2, \ldots)$ homotopic to $f$ with $E\left(f_{l}\right) \leq \rho^{l} E(f)$. But $0<\rho<1$ whence $\lim _{l \rightarrow \infty} E\left(f_{l}\right)=0$ as required.

We now prove the lemma. Choose any orthonormal basis $v_{1}, \ldots, v_{m+k}$ of $\mathbf{R}^{m+k}$ and to simplify notation set $V_{j}=v_{j}^{T}$. Because $Q_{x}^{M}$ is negative definite for all $x$ and $M$ is compact there is a constant $b>0$ such that for every vector $X$ tangent to $M$

$$
\left.\frac{1}{2} \sum_{j=1}^{m+k} \frac{d^{2}}{d t^{2}}\right|_{t=0}\left\|\varphi_{t_{*}}^{V_{j}} X\right\|^{2}=\left\langle Q^{M} X, X\right\rangle \leq-(m+k) b\|X\|^{2},
$$

where the equality follows from (3.10). This implies for any smooth $f: N \rightarrow M$ that

$$
\left.\sum_{j=1}^{m+k} \frac{d^{2}}{d t^{2}}\right|_{t=0} E\left(\varphi_{t}^{V_{j}} \circ f\right) \leq-2(m+k) b E(f) .
$$

Fix a smooth map $f: N \rightarrow M$. We now proceed in steps.

Step 1. There is a number $B \geq b>0$ such that for $1 \leq j \leq m+k,|t| \leq 1$ and all $X \in T M$

$$
\left|\frac{d^{3}}{d t^{3}}\left\|\varphi_{t_{*}}^{V_{j}} X\right\|^{2}\right| \leq B\|X\|^{2} .
$$

ProOF. Let $S M$ be the unit sphere bundle of $M$. Then the function defined on the compact set $[-1,1] \times S M$ by

$$
(t, K) \rightarrow \max _{1 \leq j \leq m+k}\left|\frac{d^{3}}{d t^{3}}\left\|\varphi_{t .}^{V_{j}} X\right\|^{2}\right|
$$

is continuous and thus has a maximum. Let $B_{0}$ be this maximum and $B=$ $\max \left\{b, B_{0}\right\}$. Then (3.14) follows by homogeneity. then

Step 2. There is a smooth vector field $V$ on $M$ such that if $B$ is as in Step 1,

$$
\begin{gathered}
\left.\frac{d}{d t}\right|_{t=0} E\left(\varphi_{t}^{V} \circ f\right) \leq 0, \\
\left.\frac{d^{2}}{d t^{2}}\right|_{t=0} E\left(\varphi_{t}^{V} \circ f\right) \leq-2 b E(f), \\
\left|\frac{d^{3}}{d t^{3}} E\left(\varphi_{t}^{V} \circ f\right)\right| \leq B E(f) \quad \text { for }|t| \leq 1 .
\end{gathered}
$$

Proof. From (3.13) it is seen that

$$
\left.\frac{d^{2}}{d t^{2}}\right|_{t=0} E\left(\varphi_{t}^{V_{j}} \circ f\right) \leq-2 b E(f)
$$


for some $j$. If $\left.(d / d t)\right|_{t=0} E\left(\varphi_{t}^{V_{j}} \circ f\right) \leq 0$ set $V=V_{j}$; otherwise, set $V=-V_{j}$. Then (3.15) and (3.16) hold. Now (3.17) follows by differentiating $E\left(\varphi_{t}^{V_{j}} \circ f\right)$ under the integral and using (3.14).

Step 3. Let $c=b / B$ (so $c \leq 1$ as $b \leq B), \rho=\left(1-\frac{1}{2} b c^{2}\right)$, and $V$ be as in Step 2. Then $0<\rho<1$ and

$$
E\left(\varphi_{c}^{V} \circ f\right) \leq \rho E(f) .
$$

Proof. Let $E(t)=E\left(\varphi_{t}^{V} \circ f\right)$. Then by Step 2 for $0 \leq t \leq c$

$$
E^{\prime \prime}(t)=E^{\prime \prime}(0)+\int_{0}^{t} E^{\prime \prime \prime}(s) d s \leq(-2 b+B c) E(f)=-b E(f) .
$$

Thus

and

$$
E^{\prime}(t)=E^{\prime}(0)+\int_{0}^{t} E^{\prime \prime}(s) d s \leq 0-b t E(f)
$$

$$
E(c)=E(0)+\int_{0}^{c} E^{\prime}(s) d s \leq\left(1-\frac{b c^{2}}{2}\right) E(f)=\rho E(f) .
$$

As both $E(c)$ and $E(f)$ are positive this inequality implies $\rho$ is positive.

We now prove the lemma. Let $f_{1}=\varphi_{c}^{V} \circ f$. Then $f_{1}$ is clearly homotopic to $f$ and we have just shown $E\left(f_{1}\right) \leq \rho E(f)$. This completes the proof.

\section{Proof of 2.2.2.}

4.1. Let $M$ and $\tilde{M}$ be as in 2.2 .2 and let $f: M \rightarrow \tilde{M}$ be a harmonic map. We recall the formalism needed for the second variation formula. In this we follow loosely the notation in $[\mathbf{E L}]$ except that we have a different sign convention on the curvature tensor. Denote the connection on $M$ (resp. on $\tilde{M}$ ) by $\nabla$ and $R$ (resp. $\tilde{\nabla}$ and $\tilde{R}$ ). Let $F=f^{-1} T \tilde{M}$ be the pull back of the tangent bundle of $\tilde{M}$ to $M$ by $f$ and let $D=f^{-1} \tilde{\nabla}$ be the pull back of $\tilde{\nabla}$ to $F$. To be a little more specific we identify smooth sections of $F$ with smooth vector fields along $f$, that is smooth maps $V: M \rightarrow T \tilde{M}$ such that $V(x) \in T \tilde{M}_{f(x)}$ for all $x \in M$. Then $D$ is defined by

$$
D_{X} V=\tilde{\nabla}_{d f(X)} V
$$

If $f_{t}$ is a smooth homotopy of $f$ with $f_{0}=f$, then the variation vector field of $f_{t}$ is

$$
V(x)=\left.\frac{d}{d t}\right|_{t=0} f_{t}(x)
$$

The second variation formula for the energy integral is [EL, p. 28]

$$
\left.\frac{d^{2}}{d t^{2}}\right|_{t=0} E\left(f_{t}\right)=\int_{M}\left(\left\langle D^{*} D V, V\right\rangle-\sum_{i=1}^{m}\left\langle\tilde{R}\left(V, d f\left(e_{i}\right)\right) d f\left(e_{i}\right), V\right\rangle\right) \Omega_{M} .
$$

Here $e_{1}, \ldots, e_{m}$ is a local orthonormal moving frame and $D^{*} D$ is defined by

$$
\left(D^{*} D V\right)_{x}=-\sum_{i=1}^{m}\left(D_{e_{i}} D_{e_{i}} V\right)_{x}
$$

where this time it is assumed the moving frame $e_{1}, \ldots, e_{m}$ is centered at $x$, that is $\left(\nabla e_{j}\right)_{x}=0$ for all $j$. 
In the case the homotopy $f_{t}$ is of the form $f_{t}=f \circ \varphi_{t}^{X}$ for a smooth field $X$ on $M$ the variation vector field is $V=d f(X)$ and the second variation can be rewritten in a form which is more useful to us. A calculation shows that in this case

$$
\begin{aligned}
D^{*} D V & =-\sum_{i} D_{e_{i}} D_{e_{i}}(d f(X)) \\
& =-\sum_{i=1}^{m}\left(D_{e_{i}} D_{e_{i}} d f\right)(X)-2 \sum_{i=1}^{m}\left(D_{e_{i}} d f\right)\left(\nabla_{e_{i}} X\right)-\sum_{i=1}^{m} d f\left(\nabla_{e_{i}} \nabla_{e_{i}} X\right) .
\end{aligned}
$$

By one of the Weitzenböck formulas for harmonic maps [EL, Proposition 3.3, p. 22]

$$
-\sum_{i=1}^{m}\left(D_{e_{i}} D_{e_{i}} d f\right)(X)=\sum_{i=1}^{m} \tilde{R}\left(d f(X), d f\left(e_{i}\right)\right) d f\left(e_{i}\right)-d f\left(\operatorname{Ric}^{M}(X)\right) .
$$

Using this and (4.5) in (4.3)

$$
\begin{aligned}
\left.\frac{d^{2}}{d t^{2}}\right|_{t=0} E\left(f \circ \varphi_{t}^{X}\right)= & -\int_{M}\left\langle d f\left(\operatorname{Ric}^{M}(X)\right), d f(X)\right\rangle \\
& -2 \int_{M} \sum_{i=1}^{m}\left\langle\left(D_{e_{i}} d f\right)\left(\nabla_{e_{i}} X\right), d f(X)\right\rangle \Omega_{M} \\
& -\int_{M} \sum_{i=1}^{m}\left\langle d f\left(\nabla_{e_{i}} \nabla_{e_{i}} X\right), d f(X)\right\rangle \Omega_{M} \\
= & -\int_{M} I_{1}(X) \Omega_{M}-2 \int_{M} I_{2}(X) \Omega_{M}-\int_{M} I_{3}(X) \Omega_{M},
\end{aligned}
$$

where each $I_{l}(X)$ denotes the obvious expression.

For each $v \in \mathbf{R}^{m+k}$ let $v^{T}, v^{\perp}$, etc. be as in the last section. Fix a point $x \in M$ and define three quadratic forms $\mathcal{Q}_{l}, l=1,2,3$, on $\mathbf{R}^{m+k}$ by

$$
\mathcal{Q}_{l}(v)=I_{l}\left(v^{T}\right) \quad \text { evaluated at } x \text {. }
$$

We now compute the trace of these. Choose an orthonormal basis $v_{1}, \ldots, v_{m+k}$ of $\mathbf{R}^{m+1}$ such that $v_{1}, \ldots, v_{m}$ is a basis of $T M_{x}$ and $v_{m+1}, \ldots, v_{m+k}$ is a basis of $T^{\perp} M_{x}$. Also choose a local orthonormal moving frame $e_{1}, \ldots, e_{m}$ on $M$ centered at $x$ such that $e_{i}(x)=v_{i}$ for $1 \leq i \leq m$. Then

$$
\operatorname{trace}\left(\mathcal{Q}_{1}\right)=\sum_{i=1}^{m}\left\langle d f\left(\operatorname{Ric}^{M}\left(e_{i}\right)\right), d f\left(e_{i}\right)\right\rangle .
$$

By (3.4) $\nabla_{e_{i}} v^{T}=\mathcal{A}^{v^{T}}\left(e_{i}\right)=A_{v^{\perp}} e_{i}$ whence

$$
\operatorname{trace}\left(\mathcal{Q}_{2}\right)=\sum_{j=1}^{m+k} \sum_{i=1}^{m}\left\langle\left(D_{e_{i}} d f\right)\left(A_{v_{j}^{\perp}} e_{i}\right), d f\left(f_{j}^{T}\right)\right\rangle=0
$$

as for each $j$ one of $v_{j}^{T}$ or $v_{j}^{\perp}$ is zero.

Again using $\nabla_{e_{i}} v^{T}=A_{v}^{\perp} e_{i}, \nabla_{e_{i}} e_{i}=0$ at $x$ and (3.5), we obtain

$$
\nabla_{e_{i}} \nabla_{e_{i}} v^{T}=\nabla_{e_{i}}\left(A_{v^{\perp}} e_{i}\right)=\left(\bar{\nabla}_{e_{i}} A\right)_{v^{\perp} e_{i}}-A_{h\left(e_{i}, v^{T}\right.} e_{i} .
$$


Also, using (2.6) so that $\left\langle h\left(e_{i}, v^{T}\right), v_{\alpha}\right\rangle=\left\langle A_{v_{\alpha}}, v^{T}, e^{i}\right\rangle$

$$
\sum_{i=1}^{m} A_{h\left(e_{i}, v^{T}\right)} e_{i}=\sum_{\alpha=m+1}^{m+k} A_{v_{\alpha}} \sum_{i=1}^{m}\left\langle A_{v_{\alpha}} v^{T}, e_{i}\right\rangle e_{i}=\sum_{\alpha=m+1}^{m+k} A_{v_{\alpha}}^{2} v^{T} .
$$

The last two equations can be combined to give

$$
\operatorname{trace}\left(\mathcal{Q}_{3}\right)=0-\sum_{j=1}^{m}\left\langle d f\left(\sum_{\alpha=m+1}^{m+k} A_{v_{\alpha}}^{2} v_{j}\right), d f\left(v_{j}\right)\right\rangle \text {. }
$$

Now combine (4.7), (4.9), (4.10), (4.13) and the formula (2.12) for $Q^{M}$ to get

4.2 PROPOSITION. Let $M^{m}$ be a compact Riemannian manifold isometrically immersed in $\mathbf{R}^{m+k}$ and $f: M \rightarrow \tilde{M}$ a harmonic map. Then for any orthonormal basis $v_{1}, \ldots, v_{m+k}$ of $\mathbf{R}^{m+k}$

$$
\left.\sum_{j=1}^{m+k} \frac{d^{2}}{d t^{2}}\right|_{t=0} E\left(f \circ \varphi_{t}^{v^{T}}\right)=\int_{M} \sum_{i=1}^{m}\left\langle d f\left(Q^{M} e_{i}\right), d f\left(e_{i}\right)\right\rangle \Omega_{M} .
$$

This implies 2.2.2(a) in the same way that Proposition 3.2 implied 2.2.1(a).

To prove $2.2 .2(\mathrm{~b})$ we first apply $2.2 .1(\mathrm{~b})$ to the identity map id: $M \rightarrow M$ to conclude there are maps in the homotopy class of id with arbitrarily small energy. By [EL, Lemma 5.16, p. 37] this implies 2.2.2(b).

\section{Homogeneous spaces.}

5.1. Let $M^{m}=G / H$ be a compact Riemannian homogeneous space of dimension $m$ and assume the isotropy representation of $H$ on the tangent space at the $e H \in$ $G / H$ is irreducible (we will say $G / H$ is isotropy irreducible). Let

$$
\Delta=\sum_{i=1}^{m}\left(e_{i} e_{i}-\nabla_{e_{i}} e_{i}\right)
$$

$\left(e_{1}, \ldots, e_{m}\right.$ an orthonormal moving frame on $\left.M\right)$ be the Laplace-Beltrami operator on $M$ and let $\lambda_{0}=0<\lambda_{1}<\lambda_{2}<\cdots$ be the nonzero eigenvalues of $\Delta$ on $M$, that is the real numbers $\lambda$ so that the eigenspace

$$
E(\lambda)=\{f: \Delta f+\lambda f=0\}
$$

has positive dimension. For $p \geq 1$ each of the eigenspaces $E\left(\lambda_{p}\right)$ induces the $p$ th standard immersion $\Phi_{p}$ of $G / H$ into some Euclidean space $\mathbf{R}^{l}$. This is defined as follows: Let $\varphi_{1}, \ldots, \varphi_{l}$ be an orthonormal basis of $E\left(\lambda_{p}\right)$ with respect to the $L^{2}$ inner product on $M$. Then for some $\alpha>0$ the map $\Phi_{p}: G / H=M \rightarrow \mathbf{R}^{l}$ given by

$$
\Phi_{p}(x)=\left(\alpha \varphi_{1}(x), \ldots, \alpha \varphi_{l}(x)\right)
$$

is an isometric immersion of $M$ into $\mathbf{R}^{l}$ and moreover the image of $\Phi_{p}$ is contained in the sphere $S^{l-1}(r)$ centered at the origin and of radius $r^{2}=m / \lambda_{p}$ as a minimal submanifold. We will also use that $\Phi_{p}$ is equivariant and that there is a group homomorphism $\rho$ from $G$ to the orthogonal group $O(l)$ acting on $\mathbf{R}^{l}$ such that

$$
\Phi_{p}(g x)=\rho(g) \Phi_{p}(x)
$$

for all $g \in G$. See [L, pp. 16-17] for details. 
We now compute the linear map $Q_{x}^{M}$ defined in $\S 2$ for the immersion $\Phi_{p}$. Because the immersion is equivariant the linear map $Q_{x}^{M}$ must commute with all elements of the isotropy subgroup of $G$ at $x$. But $Q_{x}^{M}$ is selfadjoint, therefore Schur's lemma implies $Q_{x}^{M}=c I$ ( $I$ is the identity on $\left.T M\right)$. Set $m+k=l$. Use this in (2.12) and take traces to get

$$
m c=\operatorname{trace}(c I)=-2 \operatorname{trace}\left(\operatorname{Ric}^{M}\right)+\sum_{\alpha=m+1}^{m+k} \operatorname{tr}\left(A_{e_{\alpha}}\right)^{2},
$$

where $e_{1}, \ldots, e_{m+k}$ is an orthonormal basis of $\mathbf{R}^{l}, e_{1}, \ldots, e_{m}$ a basis of $T M_{x}$ and $e_{m+1}, \ldots, e_{m+k}$ a basis of $T^{\perp} M_{x}$.

By definition the scalar curvature of $M$ at $x$ is

$$
\tau=\operatorname{trace}\left(\operatorname{Ric}^{M}\right)=\sum_{i, j=1}^{m}\left\langle R\left(e_{i}, e_{j}\right) e_{j}, e_{i}\right\rangle
$$

Because $M$ is a minimal submanifold of $S^{m+k-1}(r)$ if $e_{m+1}$ is chosen normal to $S^{m+k-1}(r)$ and $e_{m+2}, \ldots, e_{m+k}$ tangent to $S^{m+k-1}(r)$, then [Ch, Chapter 3] $\operatorname{tr}\left(A_{e_{m+1}}\right)^{2}=m^{2} / r^{2}=m \lambda p$ and $\operatorname{tr}\left(A_{e_{\alpha}}\right)=0, m+2 \leq \alpha \leq m+k$. Hence

$$
\sum_{\alpha=m+1}^{m+k} \operatorname{tr}\left(A_{e_{\alpha}}\right)^{2}=\frac{\lambda_{p}}{m} .
$$

The last four equations combine to give

5.2 PROPOSITION. For the pth standard immersion $\Phi_{p}$ of a compact isotropy irreducible Riemannian homogeneous space $M=G / H$ into Euclidean space,

$$
Q_{x}^{M}=\frac{1}{m}\left(m \lambda_{p}-2 \tau\right) I .
$$

This has an immediate corollary,

5.3 THEOREM. Let $M^{m}=G / H$ be a compact isotropy irreducible Riemannian homogeneous space with first eigenvalues $\lambda_{1}$ and scalar curvature $\tau$. Then the following are equivalent.

(A) $m \lambda_{1}<2 \tau$.

(B) The identity map on $M^{m}$ is unstable.

(C) $M^{m}$ is strongly unstable.

Proof. The conditions (A) and (B) were shown to be equivalent by Smith $[\mathbf{S}]$ (in fact he proves (A) and (B) are equivalent in any compact Einstein manifold). It is clear that (C) implies (B) and thus (A). Conversely if (A) holds then by Proposition 5.2 and Theorem $2.2 \mathrm{M}$ is strongly unstable.

Smith [S] made a partial list of which of the irreducible compact simply connected symmetric spaces have an unstable identity map. This list was completed by Nagano $[\mathbf{N}]$. Using this gives

5.4 COROLLARY. Let $M^{m}$ be an irreducible compact simply connected symmetric space. Then the standard metric on $M^{m}$ is strongly unstable if and only if $M^{m}$ 
is one of the following:

(A) $A$ sphere $S^{m}=S O(m+1) / S O(m)$ with $m \geq 3$.

(B) $\mathrm{Sp}(p+q) / \mathrm{Sp}(p) \times \mathrm{Sp}(q)$.

(C) $S U(2 m) / \mathrm{Sp}(m), m \geq 2$,

(D) The Cayley plane $\mathbf{C a y}^{2}=F_{4} / \operatorname{spin}(9)$.

(E) Any of the compact simply connected irreducible group manifolds other than the exceptional group $E_{8}$.

5.5 REMARK. If $M$ is a compact simply connected reducible symmetric space, then $M$ splits into a product $M=M_{1} \times \cdots \times M_{l}$ with each $M_{i}$ irreducible. By Remark 2.11 if each $M_{i}$ is strongly unstable then so is $M$.

\section{Some applications.}

6.1 PROPOSITION. Let $M$ be a compact Riemannian manifold with a strongly unstable metric. Then the first two homotopy groups $\pi_{1}(M), \pi_{2}(M)$ of $M$ vanish.

ProOF. If $\pi_{1}(M) \neq 0$ then it is well known [Sp, Vol. 4, Proposition 25, p. $355]$ that any nontrivial free homotopy class contains a geodesic $\gamma$ of least length and thus by the Cauchy Schwarz inequality $\gamma$ also has least energy in its homotopy class. This implies that there is a stable harmonic map from the circle $S^{1}$ into $M$. But this is impossible as $M$ is strongly unstable. Likewise if $\pi_{2}(M) \neq 0$, then by results of Sacks and Uhlenbeck [SU, Theorem 5.5, p. 22] there is at least one nonconstant stable harmonic map from $S^{2}$ into $M$. Again this is impossible as $M$ is strongly unstable.

REMARK. Recently Brian White $[\mathbf{W}]$ has shown that every map from a compact manifold into $M$ is homotopic to a map of arbitrarily small energy if and only if $\pi_{1}(M)=\pi_{2}(M)=0$.

6. 2 Proposition. Let $M^{m}=G / H$ be a compact isotropy irreducible Riemannian homogeneous space. If $m \lambda_{1}<2 \tau$, then $\pi_{1}(M)=\pi_{2}(M)=0$ and if $\lambda_{p} \neq 0$ is any eigenvalue of $M$ with $m \lambda_{p}<2$, then the pth standard immersion of $M$ into Euclidean space is an imbedding.

Proof. The vanishing of $\pi_{1}(M)$ and $\pi_{2}(M)$ follows from the last Proposition and Theorem 5.3. Assume $m \lambda_{p}<2 \tau$ but that the immersion $\Phi_{p}$ is not an imbedding. Let $M_{1}=\Phi_{p}[M]$ be the image of $M$ under $\Phi_{p}$. Then the equivariance of $\Phi_{p}$ forces it to be a covering map. Thus $M_{1}$ has a nontrivial covering manifold and therefore $M_{1}$ is not simply connected. But the local geometry of $M_{1}$ in the ambient Euclidean space is the same as that of $M$ so $M_{1}$ and $M$ have the "same" Weingarten maps. Thus

$$
Q^{M_{1}}=Q^{M}=\frac{1}{m}\left(m \lambda_{p}-2 \tau\right) I .
$$

This implies $Q^{M_{1}}$ is negative definite and so $M_{1}$ has a strongly unstable metric. But this would imply $M_{1}$ is simply connected, a contradiction. This completes the proof.

\section{REFERENCES}

[Ch] B.-Y. Chen, Geometry of submanifolds, Dekker, New York, 1973.

[EL] J. Eells and L. Lemaire, Selected topics in harmonic maps, CBMS Regional Conf. Ser. in Math., no. 50, Amer. Math. Soc., Providence, R.I., 1983. 
[ES] J. Eells and J. H. Sampson, Harmonic mappings of Riemannian manifolds, Amer. J. Math. 86 (1964), 109-160.

[L] H. B. Lawson, Lectures on minimal submanifolds, Vol. 1, Publish or Perish, Berkeley, Calif., 1980.

[Lg] P. F. Leung, On the stability of harmonic maps, Lecture Notes in Math., vol. 949, Springer, Berlin, Heidelberg and New York, 1982, pp. 122-129.

[LS] H. B. Lawson and J. Simons, On stable currents and their application to global problems in real and complex geometry, Ann. of Math. (2) 98 (1973), 427-450.

[M] Min-Oo, Maps of minimum energy from compact simply-connected Lie groups, Annals of Global Analysis and Geometry, Vol. 2, No. 1.

[N] T. Nagano, Stability of harmonic maps between symmetric spaces, Lecture Notes in Math., vol. 949, Springer, Berlin, Heidelberg and New York, 1982, pp. 130-137.

[S] R. T. Smith, The second variation formula for harmonic mappings, Proc. Amer. Math. Soc. 47 (1975), 229-236.

[Sp] M. Spivak, A comprehensive introduction to differential geometry, Publish or Perish, Berkeley, Calif., 1979.

[SU] J. Sacks and K. Uhlenbeck, The existence of minimal immersions of 2-spheres, Ann. of Math. (2) 113 (1981), 1-24.

[W] B. White, Infima of energy functionals in homotopy classes of mappings, Preprint.

$[\mathrm{X}]$ Y. L. Xin, Some results on stable harmonic maps, Duke Math. J. 47 (1980), 609-613.

Department of Mathematics, University of South Carolina, Columbia, SOUTH CAROLINA 29208 (Current addresss of Ralph Howard)

Department of Mathematics, University of CAlifornia, Los ANGeles, CaliFORNIA 90024

Current address (S. W. Wei): Department of Mathematics, University of Oklahoma, Norman, Oklahoma 73019 